\title{
Riboswitches: from ancient gene-control systems to modern drug targets
}

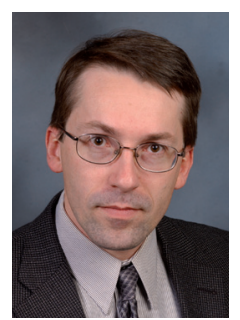

"....it has proven possible to target riboswitches with metabolite analogs to artificially modulate gene expression and disrupt bacterial growth."

\section{Ronald R Breaker}

Department of Molecular, Cellular \& Developmental Biology and Department of Molecular Biophysics \& Biochemistry and Howard Hughes Medical Institute, Yale University, 266 Whitney Avenue, New Haven CT 06520-8103, USA = Tel.: +1 2034329389 = Fax: +1 2034326161 m ronald.breaker@yale.edu

Running an efficient metabolism requires finely tuned regulatory systems to make sure that the correct metabolites are produced at the necessary times and in the appropriate concentrations. To achieve this, cells make use of numerous molecular sensors and switches to control the production and function of enzymes. Interestingly, not all small-molecule sensors are made of proteins. Many bacteria and some species of archaea and eukaryotes are known to make use of metabolitebinding gene-control elements made of RNA, called riboswitches [1,2]. These RNAs are nature's versions of small-molecule-binding aptamers, which are engineered RNAs created using methods first described by Gold [3] and Szostak [4] in the early 1990s.

Most riboswitches reside in mRNAs, where they form selective binding pockets or aptamers for their target metabolites without the requirement for protein factors. Folding changes brought about by RNA-metabolite complex formation subsequently modulate the level of expression of a protein-coding region usually located immediately downstream. At least 20 distinct classes of riboswitches have been reported since the first biochemical and genetic validation studies of riboswitches were published in 2002 [5-8], revealing their widespread distribution and utility in bacteria.

Over the past several years, I have had many discussions with other researchers regarding these riboswitch discoveries, ranging from speculations on the nature of primordial organisms to the possible applications of riboswitches as designer gene-control elements and novel drug targets. Initially it may seem frivolous for molecular engineers or drug developers to think about what life forms may have looked like shortly after the origin of life. Humans, other animals and our distant plant relatives, which our medical and agricultural institutions strive to keep healthy or genetically alter, are the products of billions of years of evolution. The elapsed eons of natural selection certainly must have changed or erased much of what was important to our long-extinct ancestors. Even our infectious adversaries, viruses, bacteria, fungi and other disease-causing organisms, have been subjected to similar evolutionary forces. The rapid rate of bacterial reproduction and adaptation compared with humans has probably allowed these organisms to put even greater evolutionary distances between them and their earliest forbearers.

However, it is apparent that some biological components and processes that are of fundamental importance to modern cells do have ancient origins. Ribosomes, for example, are RNA-protein machines that have been manufacturing polypeptides since the last common ancestor of all modern cells. Intriguingly, the catalytic core of each ribosome is made entirely of RNA [9] and, therefore, constitutes a ribozyme. Even metabolic pathways and the coenzymes that are used by enzymes as tools to catalyze these reactions are similar or identical in species from all three domains of life. In 1976, White pointed out that many coenzymes carry nucleotide fragments or are chemically derived from RNA nucleotides [10]. These observations, and many others like them, support the theory that an RNA world existed, which was populated with organisms that made little or no use of proteins and DNA [11-14].

Each new discovery of a noncoding RNA gives us another opportunity to speculate on its origin. Is this newfound RNA a relic from the RNA world? Or did the RNA emerge more recently, perhaps long after 'modern-style' DNA genomes and protein-catalyzed metabolic pathways were in place? In the late 1980s, Benner and coworkers

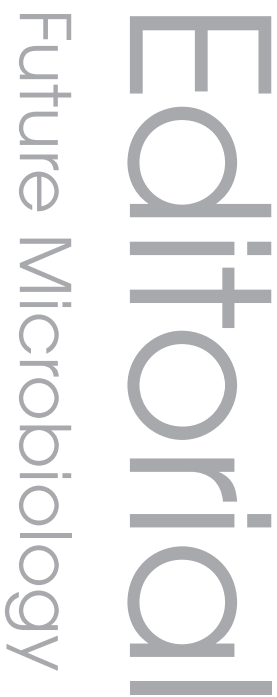

"These observations ... support the theory that an RNA world existed, which was populated with organisms that made little or no use of proteins and DNA."

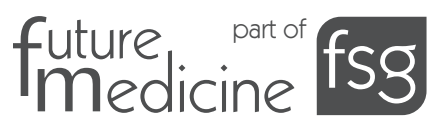


"... it is not surprising that broad-spectrum activity is a characteristic of many antibiotics that target these RNAs." published a compelling approach to help assess whether a particular RNA had its origins in or near the RNA world $[15,16]$. If homologs of a large functional RNA are nearly universally present in cells from all three domains of life, then the last common ancestor of modern organisms may have performed the same function with an ancestral RNA. By this criterion, rRNA, RNase P and signal recognition particle RNA, among others, appear to be very ancient.

The widely shared, distinctive characteristics of bacterial ribosomes have been exploited by both nature and antibiotics discovery teams to yield a variety of compounds that kill bacteria by disrupting normal protein translation. Given the fundamental importance of ribosomes in protein synthesis, and given the exceptional level of conservation of rRNAs, it is not surprising that broad-spectrum activity is a characteristic of many antibiotics that target these RNAs. Of course, since humans also carry rRNAs that share some nucleotides and structures with rRNAs from all other species, ribosome-targeting drugs must be tailored to avoid disrupting protein synthesis in the host. Moreover, the evolutionary heritage between mitochondria and bacteria creates another challenge for drug developers, since their rRNAs are even more closely related.

Not every functional RNA in modern cells can be considered a molecular relic from an RNA world. Some biochemical functions could be particularly well suited for RNA to carry out, and therefore could have emerged more recently in evolution. Although the large self-splicing ribozymes could be direct descendants from the RNA world, splice-site recognition is easily achieved without proteins simply by using Watson-Crick base pairing. Also, phosphoester transfer is the most common reaction catalyzed by known ribozymes, implying that this reaction is quite easy for active sites made of RNA to catalyze. Although self-splicing ribozymes may have found use in an RNA world, modern self-splicing RNAs could have emerged later in evolution because they may represent a more refined answer than one involving site-specific cleavage and ligation reactions catalyzed by protein enzymes.

Similar points can be made to support the hypothesis that some riboswitch classes are extremely ancient, while others are from a more recent vintage [17]. Many of the known riboswitch classes respond to coenzymes or other fundamental metabolites that likely originated in the RNA world. Furthermore, some of these riboswitch classes are structurally complex, exceedingly widespread, and well conserved in bacteria. Representatives of one of these common riboswitch classes that sense the coenzyme thiamin pyrophosphate are found in organisms from all three domains of life [18]. By contrast, some riboswitches are small, exceedingly rare and very narrowly distributed, implying that they may have emerged more recently in evolution.

Since riboswitches have evolved to selectively bind small molecules and frequently control the expression of critical metabolic pathways, it has proven possible to target riboswitches with metabolite analogs to artificially modulate gene expression and disrupt bacterial growth [19-23]. Furthermore, since the ligand-binding pockets of these RNAs must bind a compound that never changes in evolution, their core sequences are strikingly well conserved. Therefore, an analog that is designed to hit one riboswitch from a given class will likely also bind to the vast majority of the other representatives of the same class. The dearth of nucleotide diversity means that riboswitches challenged with an analog may not be able to avoid being misregulated because mutations to the binding pocket or elsewhere may prevent the natural metabolite from binding and triggering normal gene regulation.

From a drug development perspective, riboswitch vintage could be an important issue. Some of the most widespread riboswitches bind coenzymes that are essential for metabolism and they control the expression of genes whose protein products make or import more of the coenzyme. These riboswitches are candidate targets for more broad-spectrum antibiotics development. By contrast, although some of the rare riboswitch classes still sense important metabolites, their narrower distribution would permit the development only of narrow-spectrum drugs.

Furthermore, ancient riboswitches may have flourished through evolution, in part because they have binding pockets that are difficult to trick with antimetabolites. Just as some modern bacteria produce metabolite analogs in an effort to kill their competitors, ancient organisms would have done the same and may have found the earliest riboswitch classes to be inviting targets. Constant assaults on riboswitches by competitors' natural metabolite analogs would have favored the evolutionary survival of aptamers that bind ligands more selectively. Alternatively, organisms under assault may use riboswitches in genetic circuits that are more tolerant of a competitor's attempts to disrupt normal regulation.

At least one example of riboswitch-mediated chemical warfare appears to still persist. Roseoflavin, a natural analog of riboflavin that 
is biosynthesized by Streptomyces davawensis, has been shown to repress gene expression controlled by riboswitches for the coenzyme flavin mononucleotide $[22,23]$. The added dimethylamino group present in roseoflavin is accommodated by space in the binding pocket of flavin mononucleotide riboswitches, and an atomic-resolution structure model for this RNA-ligand complex [24] reveals more opportunities to exploit this pocket with additional analog designs. Although evolutionarily younger riboswitches could be even more susceptible to targeting by analogs, even the ancient riboswitches appear to have binding pockets that are vulnerable to exploitation by drug developers.

Despite the fact that there are very few classes of drugs whose mode of action involves RNA binding, the widespread use of antibiotics that target ribosomes proves that structured RNAs can be a good target. As riboswitches have naturally evolved to bind small molecules and control the expression of critical genes, they also appear to be tractable targets for drug development. Furthermore, the pace of novel noncoding RNA discovery is continuing to increase and, therefore, additional opportunities to broaden the list of RNA targets for future drug-discovery programs are likely to arise.

\section{Financial \& competing interests disclosure \\ Support for RNA science in the Breaker laboratory is received from the Howard Hughes Medical Institute and from grants supported by the NIH. The author is a cofounder of BioRelix, a biotechnology company that is pursuing the development of antibiotics that target riboswitches. The author has no other relevant affili- ations or financial involvement with any organization or entity with a financial interest in or financial con- flict with the subject matter or materials discussed in the manuscript apart from those disclosed. \\ No writing assistance was utilized in the production of this manuscript.}

\section{Bibliography}

1. Montange RK, Batey RT: Riboswitches: emerging themes in RNA structure and function. Annu. Rev. Biophys. 37, 117-133 (2008).

2. Roth A, Breaker RR: The structural and functional diversity of metabolite-binding riboswitches. Annu. Rev. Biochem. 78, 305-334 (2009).

3. Tuerk C, Gold L: Systematic evolution of ligands by exponential enrichment: RNA ligands to bacteriophage T4 DNA polymerase. Science 249, 505-510 (1990).

4. Ellington AD, Szostak JW: In vitro selection of RNA molecules that bind specific ligands. Nature 346, 818-822 (1990).

5. Nahvi A, Sudarsan N, Ebert MS, Zou X, Brown KL, Breaker RR: Genetic control by a metabolite binding mRNA. Chem. Biol. 9, 1043-1049 (2002).

6. Winkler W, Nahvi A, Breaker RR: Thiamine derivatives bind messenger RNAs directly to regulate bacterial gene expression. Nature 419, 952-956 (2002).

7. Mironov AS, Gusarov I, Rafikov R et al:: Sensing small molecules by nascent RNA: a mechanism to control transcription in bacteria. Cell 111, 747-756 (2002).

8. Winkler WC, Cohen-Chalamish S, Breaker RR: An mRNA structure controls gene expression by binding FMN. Proc. Natl Acad. Sci. USA 99, 15908-15913 (2002).

9. Steitz TA, Moore PB: RNA, the first macromolecular catalyst: the ribosome is a ribozyme. Trends Biochem. Sci. 28, 411-418 (2003).
10. White HB 3rd: Coenzymes as fossils of an earlier metabolic state. J. Mol. Evol. 7, 101-104 (1976).

11. Woese C: The Genetic Code. Harper and Row, NY, USA (1967).

12. Crick FH: The origin of the genetic code. J. Mol. Biol. 38, 367-379 (1968).

13. Orgel LE: Evolution of the genetic apparatus. J. Mol. Biol. 38, 381-393 (1968).

14. Gilbert W: Origin of life: the RNA world. Nature 319, 618 (1986).

15. Benner SA, Allemann RK, Ellington AD et al.: Natural selection, protein engineering, and the last riboorganism: rational model building in biochemistry. Cold Spring Harb. Symp. Quant. Biol. 52, 53-63 (1987).

16. Benner SA, Ellington AD, Tauer A: Modern metabolism as a palimpsest of the RNA world. Proc. Natl Acad. Sci USA 86, 7054-7058 (1989).

17. Breaker RR: Riboswitches and the RNA world. In: The RNA World (3rd Edition). Gesteland RF, Cech TR, Atkins JF (Eds). Cold Spring Harbor Laboratory Press, NY, USA, 89-107 (2006).

18. Sudarsan N, Barrick JE, Breaker RR: Metabolite-binding RNA domains are present in the genes of eukaryotes. RNA 9, 644-647 (2003).

19. Blount KF, Breaker RR: Riboswitches as antibacterial drug targets. Nat. Biotechnol. 24, 1558-1564 (2006).

20. Sudarsan N, Cohen-Chalamish S, Nakamura S, Emilsson GM, Breaker RR: Thiamine pyrophosphate riboswitches are targets for the antimicrobial compound pyrithiamine. Chem. Biol. 12, 1325-1335 (2005).

21. Blount KF, Wang JX, Lim J, Sudarsan N, Breaker RR: Antibacterial lysine analogs that target lysine riboswitches. Nat. Chem. Biol. 3, 44-49 (2006).

22. Lee ER, Blount KF, Breaker RR: Roseoflavin is a natural antibacterial compound that binds to FMN riboswitches and regulates gene expression. RNA Biol. 6, 187-194 (2009).

23. Ott E, Stolz J, Lehmann M, Mack M: The RFN riboswitch of Bacillus subtilis is a target for the antibiotic roseoflavin produced by Streptomyces davawensis. RNA Biol. (2009) (Epub ahead of print).

24. Serganov A, Huang L, Patel DJ: Coenzyme recognition and gene regulation by a flavin mononucleotide riboswitch. Nature 458, 233-237 (2009).

\section{Affiliation}

- Ronald R Breaker Department of Molecular, Cellular \& Developmental Biology and

Department of Molecular Biophysics \& Biochemistry

$$
\text { and }
$$

Howard Hughes Medical Institute, Yale University, 266 Whitney Avenue, New Haven, CT 06520-8103, USA

Tel.: +1 2034329389

Fax: +1 2034326161

ronald.breaker@yale.edu 